\title{
lncRNAs Are Involved in Sevoflurane Anesthesia-Related Brain Function Modulation through Affecting Mitochondrial Function and Aging Process
}

\author{
Yinyin Qu, ${ }^{1}$ Hongyi Li, ${ }^{2}$ Chengmei Shi $\mathbb{D},{ }^{1}$ Min Qian, ${ }^{1}$ Ning Yang $\mathbb{D},{ }^{1}$ Liwei Wang, \\ Xingyu Gao $\mathbb{D}^{3},{ }^{3}$ and Cheng $\mathrm{Ni} \mathbb{1}^{2}$ \\ ${ }^{1}$ Department of Anesthesiology, Peking University Third Hospital, Beijing 100191, China \\ ${ }^{2}$ Department of Anesthesiology, National Cancer Center/National Clinical Research Center for Cancer/Cancer Hospital, \\ Chinese Academy of Medical Sciences and Peking Union Medical College, Beijing 100021, China \\ ${ }^{3}$ Institute of Microelectronics, Chinese Academy of Sciences, Beijing 100029, China
}

Correspondence should be addressed to Xingyu Gao; gxy9910@gmail.com and Cheng Ni; nicheng@cicams.ac.cn

Received 13 September 2020; Revised 2 November 2020; Accepted 4 November 2020; Published 7 December 2020

Academic Editor: Fu-Ming Tsai

Copyright (c) 2020 Yinyin Qu et al. This is an open access article distributed under the Creative Commons Attribution License, which permits unrestricted use, distribution, and reproduction in any medium, provided the original work is properly cited.

\begin{abstract}
Long noncoding RNAs (lncRNAs) play important roles in brain function modulation and neurodegenerative diseases. However, whether lncRNA regulations are involved in the mechanisms of perioperative neurocognitive disorders, especially in anesthesiarelated brain dysfunction, remain unknown. Therefore, we explored the expression and regulation pattern profiles of lncRNAs in the hippocampus of aged rats after sevoflurane anesthesia. Three lncRNAs and 772 protein-coding genes were identified by microarray analysis and evidenced by in vitro and in vivo experiments as differentially expressed. Functional annotation and differentially expressed- (DE-) lncRNA-mRNA coexpression networks reveal that DE-lncRNAs are associated with mitochondrial dysfunction and oxidative stress, aging-related metabolism alterations, DNA damage, and apoptosis, as well as neurodegenerative features during sevoflurane anesthesia. These results suggest that lncRNAs play roles in general anesthesiarelated brain function modulation during the perioperative context and provide insights into the lncRNA-related modulation mechanisms and targets.
\end{abstract}

\section{Introduction}

Every year, 66 million senior patients aged 65 and above undergo anesthesia and surgery all around the world, including 8.5 million patients with Alzheimer's disease (AD) [1]. Up to $40 \%$ of these patients may suffer from perioperative neurocognitive disorders (PND) [2], which include postoperative delirium (POD) and postoperative cognitive dysfunction (POCD) [3]. Increasing age, preoperative cognitive impairment, surgical trauma, and anesthesia may propose the onset of PND [2, 4-6]. Aging and related neurodegenerations have features of neuroinflammation and mitochondrial dysfunction, even amyloid plaques and neurofibrillary tangles, which damage the neural processes necessary for cognition $[7,8]$. Surgery/anesthesia exposures contribute to the neuropathologic changes and cognitive decline in aged models [9]. Ours and related researches have indicated that inhaled general anesthesia is etiologically implicated in cognitive impairment in the aged brain $[10,11]$. During the process, microglial inflammation [12], mitochondrial function deficits [13], and hippocampal synaptic plasticity modulation [14] could be the contributing pathological factors. However, the role of epigenetic modulation during general anesthesiarelated brain function alterations remains unknown.

Long noncoding RNAs (lncRNAs) are noncoding RNAs with more than 200 nucleotides, which involve multiple epigenetic modulations. Increasing evidence suggests that lncRNAs play significant roles in the regulation of tissue homeostasis, oxidative stress, and metabolism $[15,16]$. In the central nervous system (CNS), the fundamental roles of $\ln$ CRNAs in various neurodegenerations are also becoming evident; representatives include BACE1-AS, NEAT1_2, and 
Meg3. These lncRNAs exert their regulation effect through various mechanisms such as interacting with mRNA (BACE1-AS) [17], binding to RNA-binding proteins (NEAT1_2) [18], or working as competing endogenous RNAs (Meg3) [19]. However, whether lncRNA regulations are involved in the mechanisms of PND, especially in general anesthesia-related brain function modulation, remain to be elucidated. Identification of candidate lncRNAs could provide insights into the mechanisms of the effects of anesthesia and efficient diagnostic strategies. Therefore, we focused on lncRNA and gene expression patterns in the hippocampus of aged rats after sevoflurane anesthesia. And we established functional annotation of differentially expressed- (DE-) lncRNAs and their potential target genes, as well as lncRNA-mRNA coexpression network to reveal the role of lncRNAs in sevoflurane anesthesia-related brain function modulation.

\section{Materials and Methods}

2.1. Animals and Anesthesia. The experiments were performed in accordance with the guide for the care and use of laboratory animals, and the protocol was approved by the Peking University Biomedical Ethics Committee Experimental Animal Ethics Branch (No. LA201412). As previously study described [20], adult male Sprague-Dawley rats, 18month old, weighing between 550 and $600 \mathrm{~g}$ were used. The rats were maintained under standard housing conditions for 2 weeks before the experiment. Food and water were provided ad libitum. The rats were randomly assigned to the sevoflurane anesthesia or control group. For whole transcriptome microarray analysis, there were 3 rats in each group. For qPCR and other experiments, there were 6 rats or 6 cell samples in each group. Previous studies revealed that the minimum alveolar concentration (MAC) of sevoflurane for rats was between $2.4-2.7 \%$ [21]. In our study, rats in the sevoflurane anesthesia group received $2.5 \%$ sevoflurane in $100 \%$ oxygen for 4 hours in an anesthetizing chamber, and the control group received $100 \%$ oxygen at an identical flow rate for $4 \mathrm{~h}$ in an identical chamber. Sevoflurane and oxygen concentrations were monitored continuously (Datex, Tewksbury, MA, USA). The rats were breathing spontaneously at a stable ambient temperature. The rectal temperatures of the animals were maintained at $37 \pm 0.5^{\circ} \mathrm{C}$. This anesthesia protocol has been shown not to significantly alter the values of blood pressure and blood gas in the preliminary studies [20]. Anesthesia was terminated by discontinuing sevoflurane and placing animals in a chamber containing $100 \%$ oxygen until 20 minutes after the recovery of consciousness. The animals were then returned to individual home cages until sacrifice. Rats were sacrificed by decapitation. The brain tissues were removed rapidly, and the hippocampus was dissected out and frozen in liquid nitrogen.

2.2. Cell Culture and Treatments. C6 rat glioma cells (CLS Cat\# 500142/p672_C6, RRID:CVCL_0194) were used in the studies. The cells were cultured in 6-well plates and grown at $37^{\circ} \mathrm{C}$ in an incubator with a humidified atmosphere with $5 \%$ $\mathrm{CO}_{2}$ in $\mathrm{F}-12 \mathrm{~K}$ medium (Gibco ${ }^{\mathrm{TM}}$, Thermo Fisher Scientific,
Waltham, MA, USA) as described in our previous study [22]. The medium was supplemented with $2.5 \%$ fetal bovine serum, $15 \%$ horse serum, $100 \mathrm{U} / \mathrm{ml}$ penicillin, and $100 \mu \mathrm{g} / \mathrm{ml}$ streptomycin. S-100 production increases ten folds as cells grow from low density to confluency, which could induce abnormal gene expression regulation in the cells. As a result, about $18 \mathrm{~h}$ after seeding, we treated the cells when they reach approximately $70 \%$ confluency. The cells were randomly assigned to a treatment or control group. In the treatment group, the cells were treated in a sealed plastic box in a $37^{\circ} \mathrm{C}$ incubator, with $4.1 \%$ sevoflurane plus $21 \% \mathrm{O}_{2}$ and $5 \% \mathrm{CO}_{2}$, delivered from an anesthesia machine for $4 \mathrm{~h}$ as described by Dong et al. [23]. The cells in the control group received vehicle gas under the same condition in the absence of sevoflurane. A Datex-infrared gas analyzer (Puritan-Bennett, Tewksbury, MA) was used to continuously monitor the delivered concentrations of carbon dioxide, oxygen, and sevoflurane. Then, the cells were harvested and frozen in liquid nitrogen.

2.3. RNA Extraction and Quantification. Total RNAs were isolated from the hippocampi and cells using TRIzol reagent (Invitrogen, Carlsbad, CA), then digested with RNase-Free DNase to remove residual DNAs. The RNA concentrations were analyzed using the Nanodrop 2000 (Thermo Fisher Scientific), then total RNA ( $2 \mu \mathrm{g}$ ) was reverse-transcribed using the GoScript ${ }^{\mathrm{TM}}$ Reverse Transcription System (Promega).

2.4. Quantitative Real-Time PCR ( $q R T-P C R)$. We performed qRT-PCR in a total reaction volume of $10 \mu \mathrm{l}$, including PowerUp SYBR ${ }^{\circledR}$ Green master mix (Thermo Fisher Scientific), $10 \mu \mathrm{M}$ PCR forward and reverse primers (Invitrogen, Carlsbad, CA, USA), and approximately $1.5 \mu \mathrm{l}$ of cDNA template on CFX96 Real-Time PCR Detection System (Bio-Rad, Hercules, CA, USA) according to the manufacturer's instructions. Primer sequences were obtained from the literature and checked for their specificity through in silico PCR. The forward and reverse primers of TC0900001760.rn.1 (NONCODE GENE ID: NONMMUG000308, Location Ch9: 36497845-9813) were 5' -AGCC CCAAAGTAAGACATTT- $3^{\prime}$ and $5^{\prime}$-CCCCTTGAGATCAC AATCAA-3', of TC1300001223.rn.1 (NONCODE GENE ID: NONMMUG001518, Location Ch13: 28298352-300631) were $5^{\prime}$-TGGTAACCAACTACTTTCGG-3' and 5'-AAACATGA GTGGAAGAGGTC-3' ${ }^{\prime}$, of TC1800000859.rn.1 (NONCODE GENE ID: NONMMUG020388, Location Ch18: 736304961258) were $5^{\prime}$-CTCCATTCTCTTACTTGAC- $3^{\prime}$ and $5^{\prime}$-CA GAGTGTACTAGGAAGCTC-3' ${ }^{\prime}$, and of TC1400000903.rn.1 (NONCODE GENE ID: NONMMUG005150, Location Ch14: 84503818-11796) were $5^{\prime}$-GACTCATTCCAGCACAGTTA$3^{\prime}$ and $5^{\prime}$-CTTGAGGGAGAATAGCAGTC-3' (These probe sets were designated according to mouse genes and located through sequence similarity alignment in rat genome). The forward and reverse primers of Hif $3 a$ were $5^{\prime}$-CACATGGACTG GGACCAAGACAGG-3' and $5^{\prime}$-GTGTAGGCTGCTGGTG TGGAGTGT- $3^{\prime}$, of Prkcd were $5^{\prime}$-CCATCTCATCTGTACC TTCC- $3^{\prime}$ and $5^{\prime}$-CCATCCTTGTCCAGCATTA- $3^{\prime}$, and of $N f e 2 l 2$ were $5^{\prime}$-GCACATCCAGACAGACACCA-3' and $5^{\prime}$-GGCTGGGAATATCCAGGGCA-3'. Amplification was 
carried out with an initial denaturation step at $95^{\circ} \mathrm{C}$ for $2 \mathrm{~min}$, followed by 45 cycles of $95^{\circ} \mathrm{C}$ for $10 \mathrm{~s}, 55^{\circ} \mathrm{C}$ for $30 \mathrm{~s}$, and $60^{\circ} \mathrm{C}$ for $30 \mathrm{~s}$, then a final extension at $65^{\circ} \mathrm{C}$ for $2 \mathrm{~min}$ in $10 \mu \mathrm{l}$ reaction volume. After amplification, a melt curve was performed to make sure that none of the nonspecific products such as primer dimers were amplified. All reactions were run in duplicate, and the results were averaged from 6 independent studies. qPCR was quantified in two steps, firstly, $A c t b$ ( $\beta$-actin) levels were used to normalize target gene levels ( $\Delta$ Cycle threshold $(\Delta \mathrm{Ct})=\mathrm{Ct}_{\text {target gene }}-\mathrm{Ct}_{\mathrm{Actb}}$, target gene level $=2^{-\Delta \mathrm{Ct}}$ ). Beta-actin was chosen as an internal control because previous studies confirmed that it was one of the most stable genes in the hippocampus of rats [24]. It also acts as a reliable endogenous control and has been widely used in the context of sevoflurane anesthesia in aged rats $[25,26]$. Secondly, the target gene levels of the sevoflurane group were presented as the percentage of those of the control group, and $100 \%$ of the target gene levels referred to the control levels.

\subsection{Affymetrix Whole Transcriptome Microarray Analysis} and Functional Annotation. Whole transcriptome microarray analysis was performed using Clariom ${ }^{\mathrm{TM}}$ D Pico Assay, previously known as GeneChip ${ }^{\mathrm{TM}}$ Rat Transcriptome Array 1.0 (Affymetrix, Santa Clara, CA, https://www.thermofisher .com/order/catalog/product/902666). This array has a full coverage of the transcribed genome, including both coding and noncoding splicing variants. Briefly, isolated RNA (100 ng) was mixed with $1.5 \mu \mathrm{l}$ of Poly-A RNA control solution and subjected to reverse transcription. The obtained cDNA was used for in vitro transcription to prepare antisense RNA (aRNA) by incubation at $40^{\circ} \mathrm{C}$ for $16 \mathrm{~h}$. Then, the aRNA was applied for the second round of sense cDNA synthesis using the WT Expression kit (Ambion, Austin, TX). The obtained cDNA was used for biotin labeling and fragmentation by Affymetrix GeneChip ${ }^{\circledR}$ WT Terminal Labeling and Hybridization. Biotin-labeled fragments of cDNA $(5.5 \mu \mathrm{g})$ were hybridized to the Affymetrix ${ }^{\circledR}$ Rat Transcriptome Array Strip $\left(45^{\circ} \mathrm{C} / 24 \mathrm{~h}\right)$, and up to 25 unique probes sequences were hybridized to a single transcript. Following hybridization, each array strip was washed and stained using the Fluidics Station of GeneChip ${ }^{\circledR}$ Scanner 3000 7G system (Affymetrix, Santa Clara, CA). The array strips were scanned using the Imaging Station of the GeneChip ${ }^{\circledR}$ Scanner $30007 \mathrm{G}$ system.

2.6. Mitochondrial Preparation. Tissue mitochondrial isolation kit (Beyotime Biotech, Shanghai, China) was used for mitochondrial preparation. The hippocampus was dissected out $3 \mathrm{~h}$ after anesthesia and placed in a freshly prepared cold mannitol solution encompassing $10 \mathrm{mM}$ HEPES-potassium hydroxide, $70 \mathrm{mM}$ sucrose, $0.1 \%(w / v)$ BSA, $200 \mathrm{mM}$ d-mannitol, and $1 \mathrm{mM}$ EDTA at $\mathrm{pH}$ of 7.4. Then, it was homogenized, the broken cell debris and nuclei were sedimentated by centrifugation at $600 \times \mathrm{g}$ and $4^{\circ} \mathrm{C}$ for $5 \mathrm{~min}$, and the supernatant was carried out after centrifugation at $10,000 \times \mathrm{g}$ and $4^{\circ} \mathrm{C}$ for $10 \mathrm{~min}$. The resulting mitochondrial pellets were suspended in the mannitol solution.
2.7. Reactive Oxygen Species (ROS) Assay. A ROS assay kit (Beyotime Biotech, Shanghai, China) with fluorescent probe DCFH-DA was used to measure the ROS level in the hippocampal mitochondria. Incubation of the hippocampal mitochondria suspensions $(0.5 \mathrm{mg}$ protein $/ \mathrm{ml})$ was carried out with $1.6 \mu \mathrm{M} \mathrm{2}$, $7^{\prime}$-dichlorofluorescin diacetate (DCFH$\mathrm{DA})$ at $37^{\circ} \mathrm{C}$ for $10 \mathrm{~min}$. The fluorescence was measured with the Perkin Elmer LS-50B Luminescence fluorescence spectrophotometer (California, USA) at the excitation and emission wavelength of 488 and $525 \mathrm{~mm}$, respectively. Then, the mean fluorescence intensities in excitation/emission were quantified and compared.

2.8. Mitochondrial Membrane Potential (MMP) Assay. An MMP assay kit (Beyotime Biotech, Shanghai, China) with fluorescent probe JC-1 was used to measure MMP of the hippocampal mitochondria. Incubation of the hippocampal mitochondria suspensions $(0.5 \mathrm{mg}$ protein $/ \mathrm{ml})$ was carried out with $\mathrm{JC}-1$ reagent at $37^{\circ} \mathrm{C}$ for $15 \mathrm{~min}$. The red fluorescence was measured at the excitation and emission wavelength of 525 and $590 \mathrm{~mm}$, and green fluorescence was measured at the excitation and emission wavelength of 490 and $530 \mathrm{~mm}$ with the Perkin Elmer LS-50B Luminescence fluorescence spectrophotometer. The level of MMP was calculated by the ratio of red fluorescence to green fluorescence.

2.9. Statistical Analysis. Statistical analysis was performed with GraphPad Prism 5.0 software (http://graphpad.com, RRID: SCR_002798). The quantitative data are presented as the mean $\pm \overline{S D}$. In vivo and in vitro PCR validations were displayed with the violin plots, which depict the kernel probability density, and the width of the shaded area shows the proportion of the data located at that expression fold. Unpaired two-tailed Student's $t$-test was used to determine significant difference between the two groups. One-way ANOVA with Bonferroni's multiple comparison test was used to analyze significant differences between multiple groups. $p<0.05$ was considered significant. The microarray analysis was performed by Expression Console Transcriptome Analysis Console Software. One-way ANOVA was applied. $p$ value was adjusted with FDR method (Benjamini-Hochberg procedure). DE-lncRNAs were screened with $p<0.05$ and $\mid$ fold change $\mid>1$.5. Unsupervised twoway hierarchical clustering of DE-lncRNAs was illustrated in the heat map. The significance of GO and KEGG enrichment was calculated by the hypergeometric distribution and Fisher exact test, and a lower $p$ value indicated that the specific term was more significantly enriched.

\section{Results}

The aged rats were randomly assigned to sevoflurane and control groups. The sevoflurane group received $4 \mathrm{~h}$ sevoflurane anesthesia, and the hippocampus was dissected out $3 \mathrm{~h}$ after anesthesia. In the hippocampus, 25204 lncRNAs were identified by whole transcriptome microarray analysis (Clariom $^{\mathrm{TM}}$ D Pico Assay), which allows transcriptome profiling of both coding and noncoding genes using multiple databases. Since the functions and related mechanisms of lncRNAs 
could be speculated according to the classification based on the locations relative to protein-coding genes in the genome, the classification of these lncRNAs was analyzed. The results showed that 9654 lncRNAs (38.3\%) were intergenic, 3620 lncRNAs (14.4\%) were antisense, 3287 lncRNAs (13.0\%) were sense no exonic, 50 lncRNAs $(0.20 \%)$ were exonic, 11 lncRNAs (0.04\%) were intronic, and 8582 lncRNAs (34.1\%) were others (Figure 1(a)).

The expression level of lncRNAs in the sevoflurane group versus that in the control group was presented as a BlandAltman plot based on the microarray analysis, and $514 \mathrm{DE}-$ lncRNAs were found and highlighted in red $(p<0.05$, Figure 2(a)). Hierarchical cluster analysis showed a clear distinction in expression values of the DE-lncRNAs, in which 232 lncRNAs were found to be upregulated, and 282 lncRNAs were downregulated in the sevoflurane group (Figure 2(b)). Based on the abovementioned classification based on the genomic locations, the numbers of upregulated lncRNAs belonging to intergenic, antisense, sense no exonic, exonic, intronic, and others categories were 88, 31, 29, 1, 0, and 83 , respectively (Figure $1(\mathrm{~b})$ ). Meanwhile, the numbers of downregulated lncRNAs that belong to the above six categories were $81,18,62,0,0$, and 121, respectively (Figure 1(c)).

Taking |fold change $\mid>1.5$ as the cutoff, four lncRNAs were filtered out. Among them, NONMMUG000308, NONMMUG001518, and NONMMUG005150 were upregulated, and NONMMUG020388 was downregulated. These four lncRNAs all fell into the lincRNA category.

To validate the microarray analysis results of four lncRNAs (NONMMUG000308, NONMMUG001518, NONMMUG005150, and NONMMUG020388), qPCR was employed. The aged rats were also assigned to sevoflurane and control groups. The sevoflurane group received $4 \mathrm{~h}$ sevoflurane anesthesia. Given that the expression of postoperative neurotoxicity and relative RNA transcription emerged at $3 \mathrm{~h}$ after surgery and could persist for $24 \mathrm{~h}[27,28]$, the hippocampus was dissected out at $3 \mathrm{~h}$ and $24 \mathrm{~h}$ after anesthesia, respectively. Violin plots were used to illustrate the PCR results $(n=6$, Figures $3(\mathrm{a})-3(\mathrm{~h}))$. The violin plot diagrams depict the kernel probability density, and the width of the shaded area shows the proportion of the data located at that expression fold. The qPCR results were consistent with microarray results in the three candidate lncRNAs (NONMMUG000308, NONMMUG001518, and NONMMUG005150). For NONMMUG000308, the expression increased significantly at $3 \mathrm{~h}$ $(2.04 \pm 0.50$ vs. $1.00 \pm 0.44, p<0.05)$, but not at $24 \mathrm{~h}$ $(1.72 \pm 0.54, p>0.05)$ after anesthesia. For NONMMUG001518, the expression increased significantly at both $3 \mathrm{~h}$ and $24 \mathrm{~h}$ after anesthesia $(2.87 \pm 1.20$ and $2.99 \pm 1.32$ vs. $1.00 \pm 0.21, p<0.05)$. For NONMMUG005150, the expression increased significantly at both $3 \mathrm{~h}(2.78 \pm 1.25$ vs. $1.00 \pm 0.28, p<0.05)$ and $24 \mathrm{~h}(4.03 \pm 1.39, p<0.001)$ after nesthesia. However, qPCR validation did not show a significant change for NONMMUG020388 (0.98 \pm 0.29 and $1.03 \pm 0.19$ vs. $1.00 \pm 0.23, p>0.05)$. Furthermore, we performed an in vitro qPCR validation to confirm the uniformity of lncRNAs performance across a range of experimental conditions. The in vitro experiment was conducted in C6 rat glioma cells with control conditions and $4 \mathrm{~h}$ sevoflurane exposure. The results provided confirmatory evidence that NONMMUG000308 (1.98 \pm 0.84 vs. $1.00 \pm 0.45, \quad p<0.05)$, NONMMUG001518 (2.12 \pm 0.58 vs. $1.00 \pm 0.42, p<0.01)$, and NONMMUG005150 (2.48 \pm 1.05 vs. $1.00 \pm 0.42, p<$ 0.01 ) in C6 cells are significantly upregulated after sevoflurane exposure, but no significant difference of NONMMUG020388 expression were found between two groups. Thus, the qPCR validation demonstrated good consistency between in vivo and in vitro models. As data quality parameters such as array $p$ values and fold change may exert influence on the consistency of the two methods, we assume PCR validations across different experimental conditions are more reliable according to previous studies [29].

Considering the three candidate DE-lncRNAs are all lincRNAs, which can positively or negatively adjust the expression of target genes, the expression of protein-coding genes in the hippocampus of aged rats was also detected by whole transcriptome microarray analysis. 772 DE-protein coding genes were identified after sevoflurane anesthesia $(p<0.05)$ with 608 genes upregulated and 164 downregulated. Then, Gene Ontology (GO) functional annotation and Kyoto Encyclopedia of Genes and Genomes (KEGG) pathway enrichment analysis were conducted for DEprotein coding genes using Database for Annotation, Visualization, and Integrated Discovery (DAVID, https://david. ncifcrf.gov). GO enrichment analysis contains three categories: biological process, molecular function, and cellular component. Hypergeometric distribution was used to determine whether a GO term is overrepresented after anesthesia. A lower $p$ value indicated that the GO or KEGG term was more significantly enriched, and the results of DAVID GO analysis revealed that $44 \mathrm{GO}$ terms of biological process, 16 terms of molecular function, and 18 terms of cellular component were significantly enriched after sevoflurane treatment $(p<0.05)$, respectively. A bubble plot to visualize enriched GO terms is shown in Figure 4(a). GO term circle in the upper part of the figure represents a relatively lower $p$ value, while a circle with larger diameter indicates more genes were involved in a specific GO term. The top significantly overrepresented terms, sorted by $p$ values in ascending order for biological process (green) were response to hypoxia, aging, cellular response to hypoxia, intracellular receptor signaling pathway, and regulation of cell cycle $(p<0.05)$; for molecular function (blue) were protein binding, RNA polymerase II transcription factor activity, identical protein binding, and Wnt-activated receptor activity $(p<0.05)$; for cellular component (red) were mitochondrion, cytosol, extracellular exosome, and cytoplasm $(p<0.05)$.

Then, KEGG pathway analysis was employed to reveal involved molecular interaction, reaction, and relation networks after sevoflurane anesthesia. Figure 4(b) highlighted 18 significantly enriched signaling pathways in our annotation using $-\log p>1$ as a threshold, including certain signaling pathways such as adipocytokine, estrogen, regulating pluripotency of stem cells, Wnt, MAPK, glucagon, and AMPK; as well as metabolic-related pathways such as metabolic pathways, lysine degradation, inositol phosphate metabolism, and biosynthesis of amino acids. Adipocytokine 


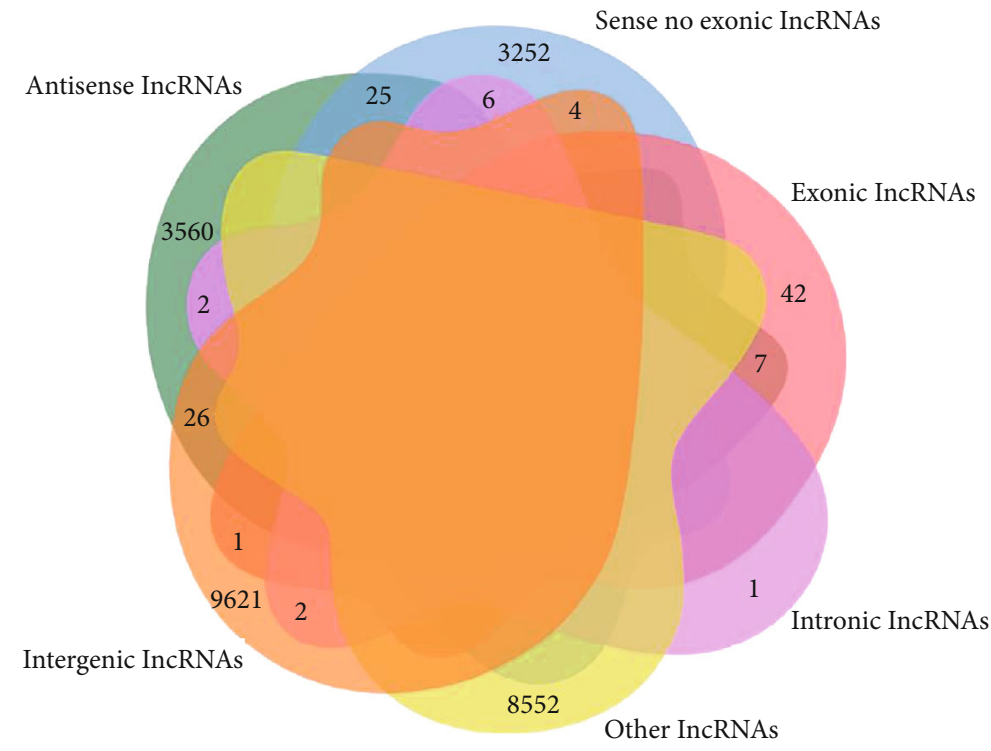

(a)

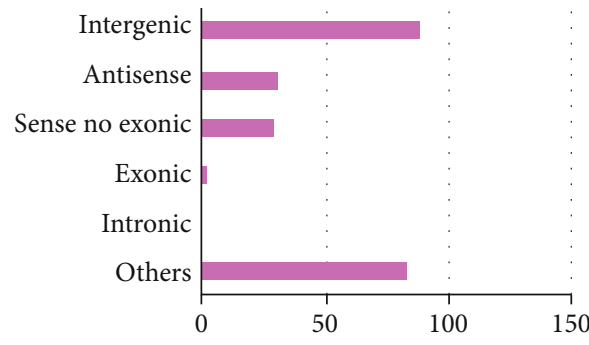

(b)

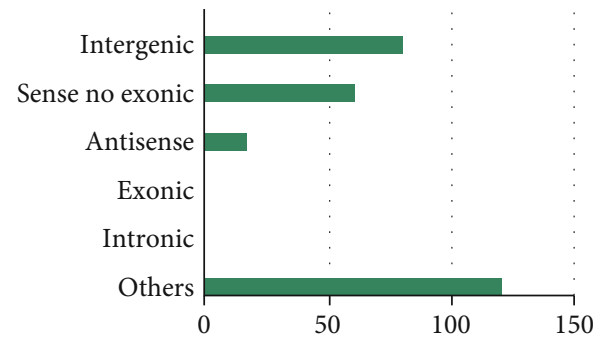

(c)

FIGURE 1: Distribution and expression of lncRNAs identified by whole transcriptome microarray analysis in the hippocampus of aged rats. (a) Venn distribution of lncRNAs. (b) Numbers of upregulated lncRNAs in different categories. (c) Numbers of downregulated lncRNAs in different categories.

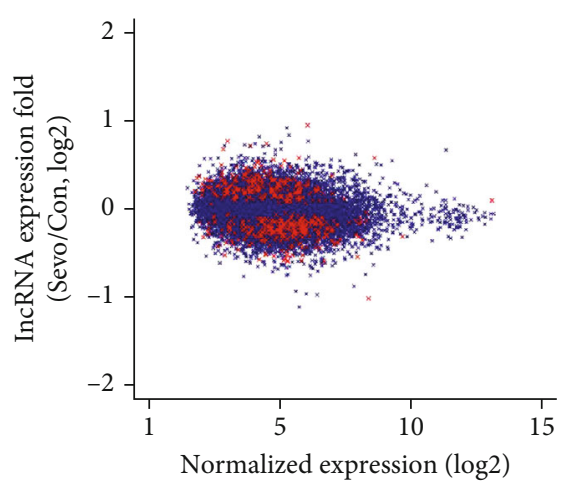

(a)

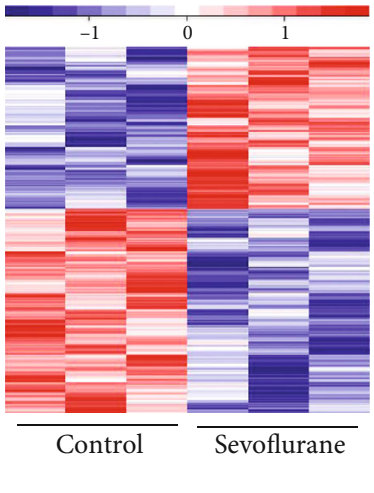

(b)

FiguRE 2: Differentially expressed lncRNAs after sevoflurane anesthesia in microarray analysis. (a) Ratio of gene expression in sevoflurane group to control condition ( $y$-axis) and average expression of genes in sevoflurane group versus that in control condition ( $x$-axis), presented as a Bland-Altman plot of our microarray analysis. Highlighted in red are lncRNAs with significant changes in expression $(p<0.05)$. (b) The heat map shows lncRNAs with significant changes from microarray analysis $(p<0.05)$.

signaling pathway was the top significantly overrepresented pathway, and term metabolic pathways were associated with the most DE genes (55 DE-genes).

The mitochondrial membrane potential (MMP) is a factor determining the viability of mitochondria, thus plays a significant role in mitochondrial homeostasis [30]. It is also a driving force for ATP synthesis, and the loss of MMP could be the sign for apoptosis [31]. Thus, the MMP was assessed, and the results showed that compared with the control group, the level of MMP significantly decreased in the 


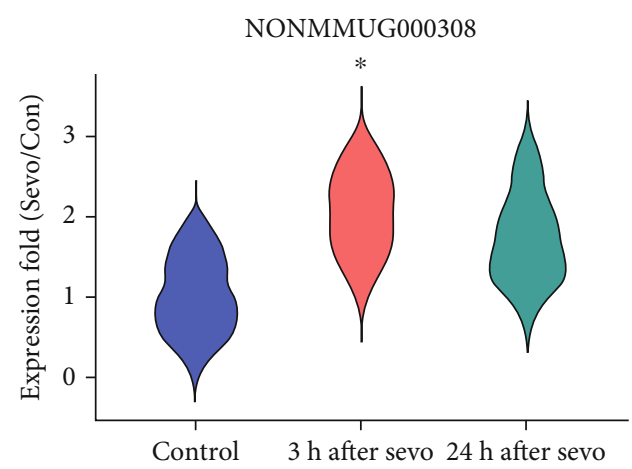

(a)

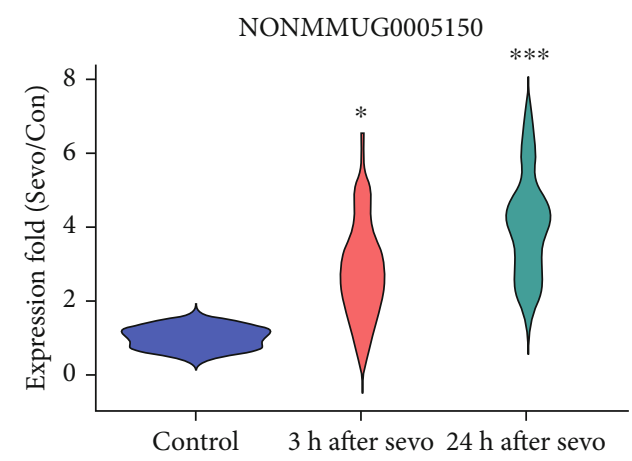

(c)

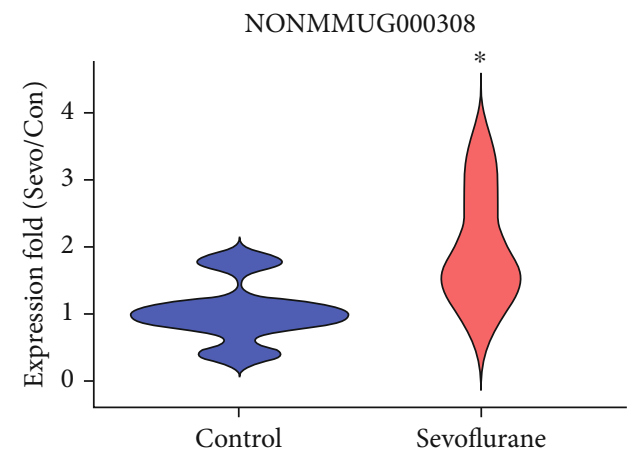

(e)

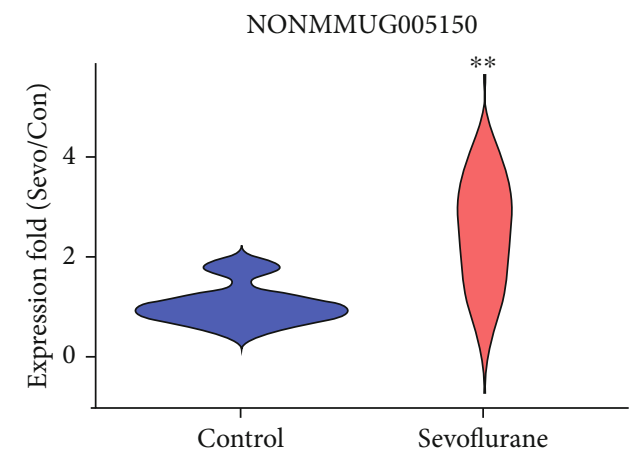

(g)

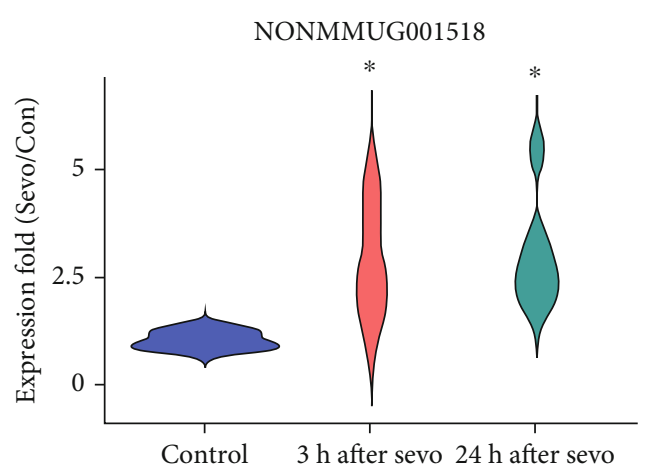

(b)

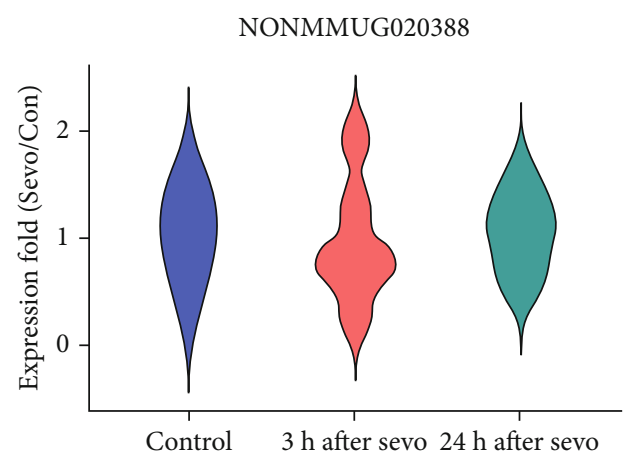

(d)

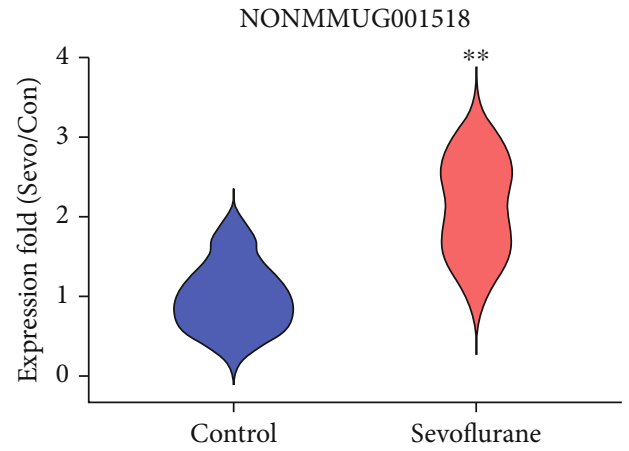

(f)

NONMMUG020388

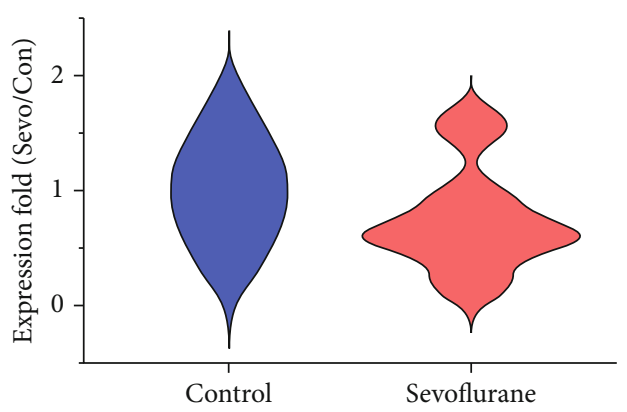

(h)

FIGURE 3: qPCR validation for differentially expressed lncRNA NONMMUG000308, NONMMUG001518, NONMMUG005150, and NONMMUG020388 in vivo $(\mathrm{a}-\mathrm{d})$ and in vitro $(\mathrm{e}-\mathrm{h})$. Gene expression was normalized and calculated using the $2^{-\Delta \Delta \mathrm{Ct}}$ method. $n=6$, ${ }^{*} p<0.05,{ }^{* *} p<0.01$, and ${ }^{* * *} p<0.001$ indicated differentiated samples compared with control condition.

sevoflurane group $(p<0.05$, Figure 4(c)). Mitochondrial dysfunction is the major cause of oxidative stress and reactive oxygen species (ROS) generation [32], so we measured the
ROS level in the hippocampus of aged rats as well. The results showed that compared with the control group, the level of ROS increased significantly in the sevoflurane group 


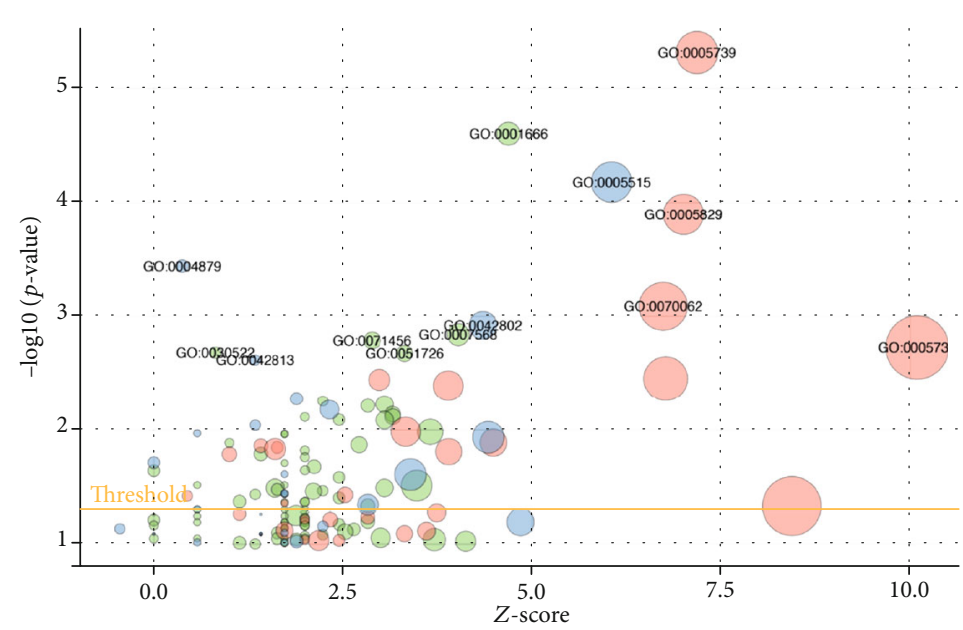

\begin{tabular}{lc}
\hline Term ID & Description \\
\hline GO:0001666 & Response to hypoxia \\
GO:0007568 & Aging \\
GO:0071456 & Cellular response to hypoxia \\
GO:0030522 & Intracellular receptor signaling pathway \\
GO:0051726 & Regulation of cell cycle \\
\hline GO:0005515 & Protein binding \\
GO:0004879 & RNA polymerase II transcription factor \\
GO:0042802 & activity \\
GO:0042813 & Wnt-activated receptor activity \\
\hline GO:0005739 & Mitochondrion \\
GO:0005829 & Cytosol \\
GO:0070062 & Extracellular exosome \\
GO:0005737 & Cytoplasm \\
\hline
\end{tabular}
Category
Biological process
Molecular function
Cellular component

(a)

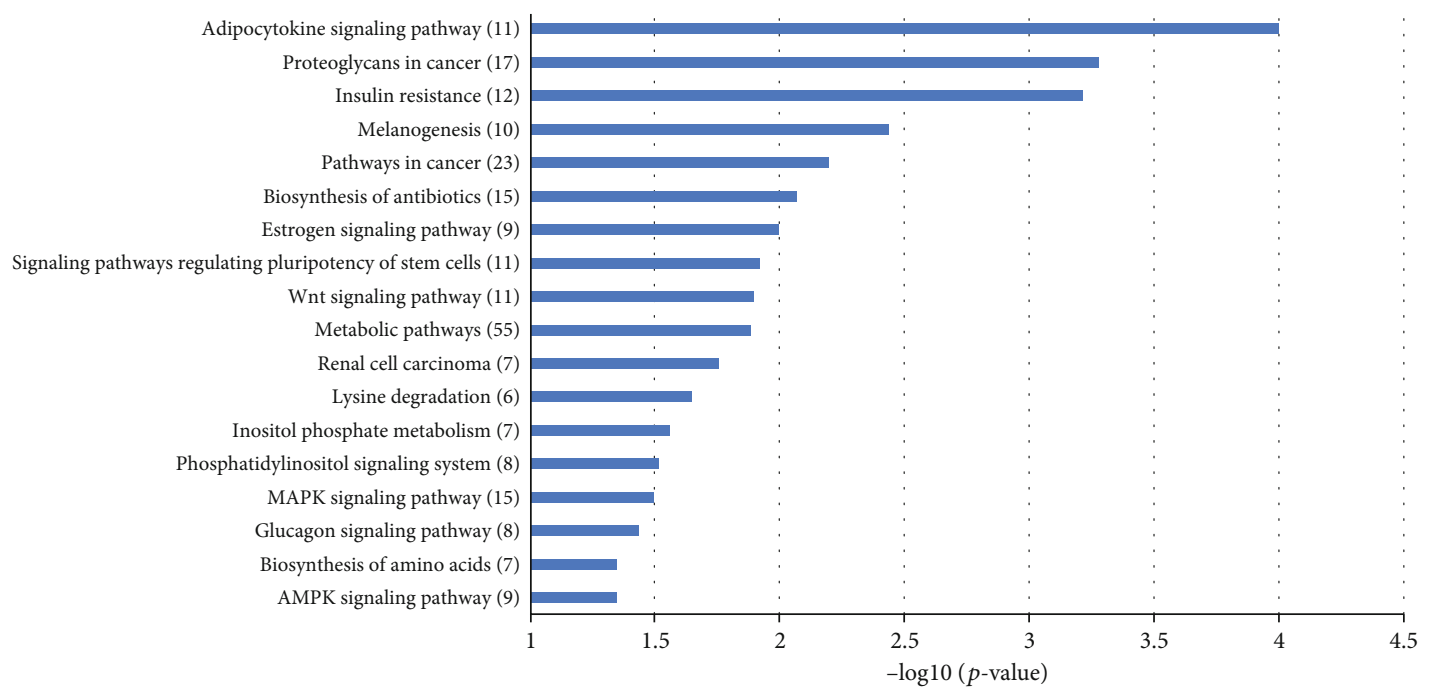

(b)

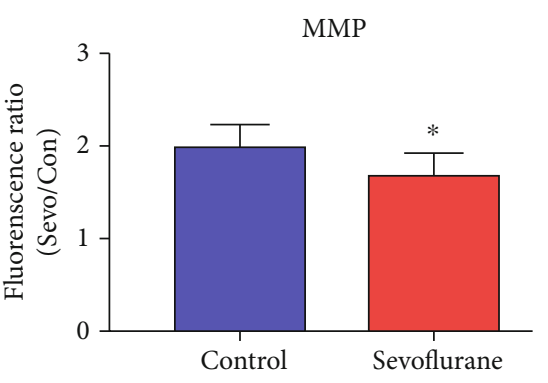

(c)

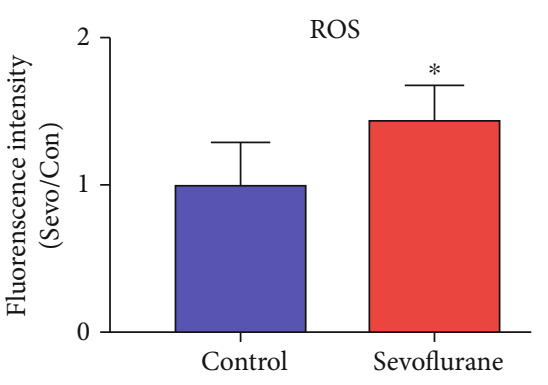

(d)

FIGURE 4: (a) Bubble plot of GO analysis of differentially expressed genes after sevoflurane anesthesia using the DAVID database. $y$-axis: the negative logarithm of $p$ value. $x$-axis: $z$ score of GO terms. The green, blue, and red bubbles indicate biological process, molecular function, and cellular component. $Z$ score $=($ up - down $) / \sqrt{ }$ count. Up is the number of assigned genes upregulated $(\log 2(\mathrm{FC})>0)$ in the data, and down is those downregulated $(\log 2(\mathrm{FC})<0)$. (b) KEGG enrichment analysis of differentially expressed genes. $y$-axis: KEGG term descriptions. The numbers of differentiated genes enriched in each KEGG term were displayed in brackets. $x$-axis: the negative logarithm of $p$ value of different KEGG terms. (c) MMP levels decreased after sevoflurane anesthesia, indicated by the ratio of red fluorescence to green fluorescence. (d) ROS levels increased after sevoflurane anesthesia, indicated by increased fluorescence intensity. ${ }^{*} p<0.05$ indicated differentiated samples compared with the control condition. 
$(p<0.05$, Figure $4(\mathrm{~d}))$. These results indicated that mitochondrial dysfunction, MMP decrease, and oxidative stress were involved in the pathophysiological processes after sevoflurane anesthesia, which are correlated with GO and KEGG analysis results.

GO enrichment analysis revealed different patterns of potential target genes of the three upregulated lncRNAs after sevoflurane anesthesia. The results were ranked according to the negative $\operatorname{logarithm} p$ value, and the terms with $-\log p>2$ of each category were displayed. For the potential target genes of NONMMUG000308, response to hypoxia, protein binding, and mitochondrion were top significant enriched terms in biological process, molecular function, and cellular component, respectively (Figure 5(a)). Protein binding is an unspecific annotation which suggested target genes may interact with proteins like p53 or with one or more sites on receptor molecules like signaling receptors. For NONMMUG001518, drug metabolic process and protein binding were the top significant enriched terms in biological process and molecular function, and top cellular component term included membrane, ribosome, and mitochondria (Figure 5(b)). This indicated that products of the target genes may be found in cellular membrane, ribosome, or mitochondria. For NONMMUG005150, cellular response to hypoxia, $\mathrm{BH}$ domain binding, and $\mathrm{Bcl}-2$ family complex were the top significant enriched terms in three categories (Figure 5(c)). Thus, potential target genes of three upregulated lncRNAs after sevoflurane anesthesia were enriched in oxidative stress, mitochondrion, metabolism, apoptosis, etc.

KEGG pathway analysis revealed the signaling pathways of potential target genes of the three DE-lncRNAs after sevoflurane anesthesia. The results were ranked according to the negative $\operatorname{logarithm}$ of $p$ value, and the terms with $-\log p>1$ were displayed. The signaling pathways of potential target genes of NONMMUG000308 and NONMMUG001518 are similar. Biosynthesis of antibiotics, signaling pathways of adipocytokine, and regulating pluripotency of stem cells, metabolic pathways were significantly enriched. Metabolic pathways including energy, carbohydrate, lipid, nucleotides, and amino acid metabolism were associated with the most potential target genes (63 DE-mRNAs, Figures 6(a) and 6(b)). For NONMMUG005150, the top enriched terms were basal cell carcinoma and Fanconi anemia pathway (Figure 6(c)). Pathways of carcinoma and anemia are characterized by abnormal proliferation and impaired response to DNA damage.

To explore the potential regulatory mechanism of the DE-lncRNAs, correlation analysis was carried out between the DE-lncRNAs and DE-protein coding genes. The DE protein-coding genes with Pearson correlation coefficient (PCC) $>0.85$ and $p<0.05$ were considered as the potential target genes of DE-lncRNAs. Pearson correlation is a traditional statistical metric widely used by previous studies to predict the function of lncRNAs by using information from coexpressed mRNAs [15, 33], and coexpressing genes are involved in related processes. Furthermore, the coexpression network of DE-lncRNAs and their potential target genes was established. Cytoscape 3.6.1 (http://www.cytoscape.org, RRID:SCR_003032) was used in network visualization, and the correlations with PCC $>0.85$ for lncRNA-mRNA (protein-coding gene) pairs and PCC $>0.95$ between mRNAmRNA (protein-coding gene) pairs were selected.

The RNAs were displayed with nodes (three DElncRNAs and 296 mRNAs). Positive coexpression represented by red edges, negative correlation represented by green edges, and lower transparency of edges mean higher PCC. 342 positive and 53 negative lncRNA-mRNA pairs were displayed. NONMMUG000308 was coexpressed with 224 mRNA transcripts, 193 upregulated and 31 downregulated. NONMMUG001518 was coexpressed with 113 mRNA transcripts, 99 upregulated and 14 downregulated. And NONMMUG005150 was coexpressed with 58 mRNA transcripts, 50 upregulated and 8 downregulated. According to the above functional annotation, DE-lncRNAs were associated with oxidative stress and mitochondrial dysfunction, metabolic pathways, DNA damage, and apoptosis after sevoflurane anesthesia. Thus, the enriched term response to hypoxia, mitochondrion, and aging (with relatively lower $p$ value) was selected as the representative terms. IncRNA coexpressed genes in these three terms were highlighted in blue, green, and yellow nodes, and other terms were highlighted in orange nodes. Genes that are highly correlated to each other are likely to be involved in the same pathway, and connections between protein-coding genes were also outlined to represent protein-protein interaction (Figure $7(\mathrm{a})$ ).

Genes from term response to hypoxia, mitochondrion, and aging with relative high PCC with at least two lncRNAs were screened out. They included Plat, Epas1, Prkcd, Agtrap, Tnfrsf $1 a, H i f 3 a$, and Tgfbr3 in term response to hypoxia; Gjb6, Prkcd, Nfel2l2, and Fgf2 in aging; as well as 16 genes (Prkcd, Oxall, etc) in term mitochondrion. Then, three representative DE-protein coding genes were selected and validated by qPCR (Hif 3 a from term hypoxia, Prkcd from term mitochondria, and $\mathrm{Nfe} 2 \mathrm{l} 2$ from term aging). Compared with control conditions, the expression of Hif $3 a(1.82 \pm 0.52$ vs. $1.00 \pm 0.21, p<0.01)$, Prkcd $(1.58 \pm 0.31$ vs. $1.00 \pm 0.44$, $p<0.05)$, and $N f e 2 l 2(1.66 \pm 0.52$ vs. $1.00 \pm 0.41, p<0.05)$ in the hippocampus increased significantly at $3 \mathrm{~h}$ after anesthesia $(n=6$, Figures $7(\mathrm{~b})-7(\mathrm{~d}))$. These results also indicated a good consistency between qPCR and microarray analysis results.

As lncRNA NONMMUG000308, NONMMUG001518, and NONMMUG005150 were differentially expressed after sevoflurane anesthesia and might play regulatory roles through oxidative stress and mitochondrial dysregulation, aging-related metabolic alterations, DNA damage, apoptosis, and neurodegenerative features according to the present results, we named NONMMUG000308, NONMMUG001518, and NONMMUG005150 as sevoflurane associated noncoding RNA (Sancr) 1, 2, and 3, respectively.

\section{Discussion}

The present study screened three significantly DE-lncRNAs and multiple DE-protein coding genes in the hippocampus of aged rats after sevoflurane anesthesia by microarray analysis. In vitro and in vivo experiments verified the analysis results. Functional annotation with GO and KEGG databases 


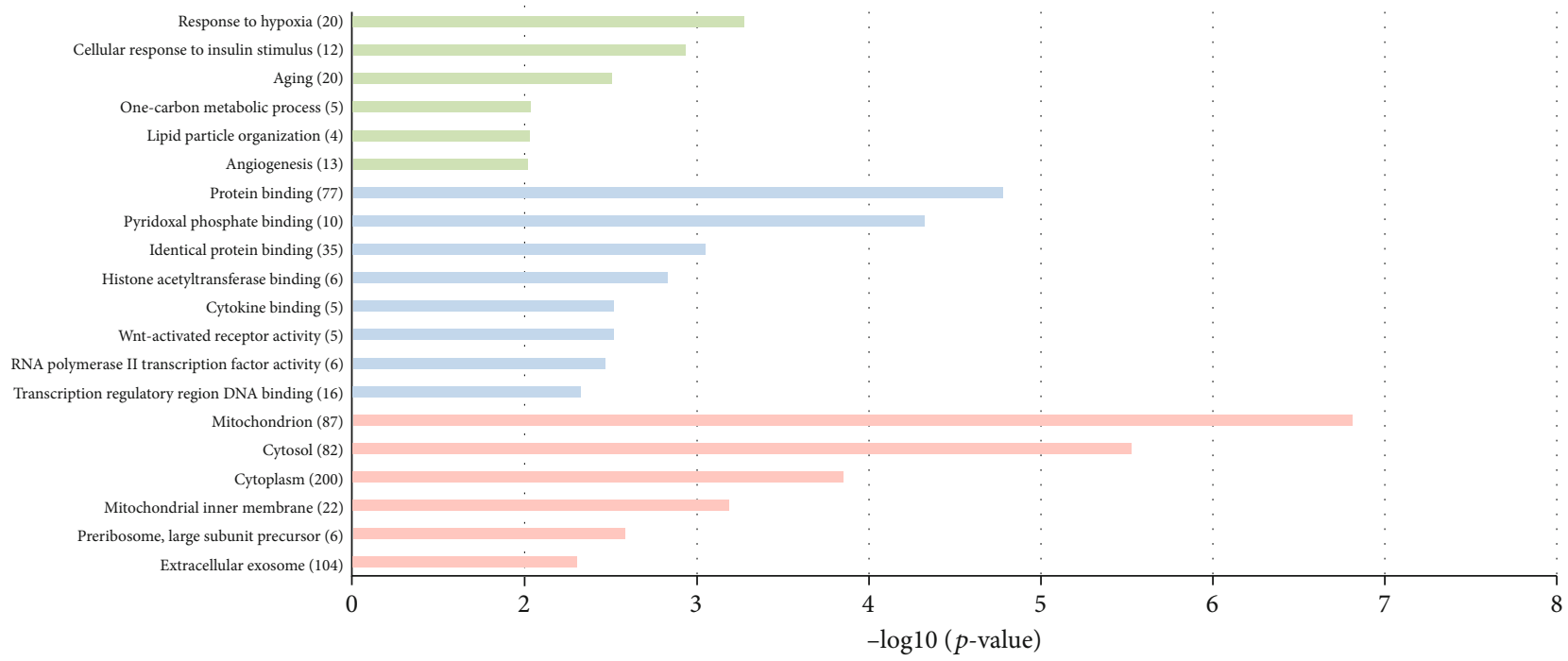

(a)

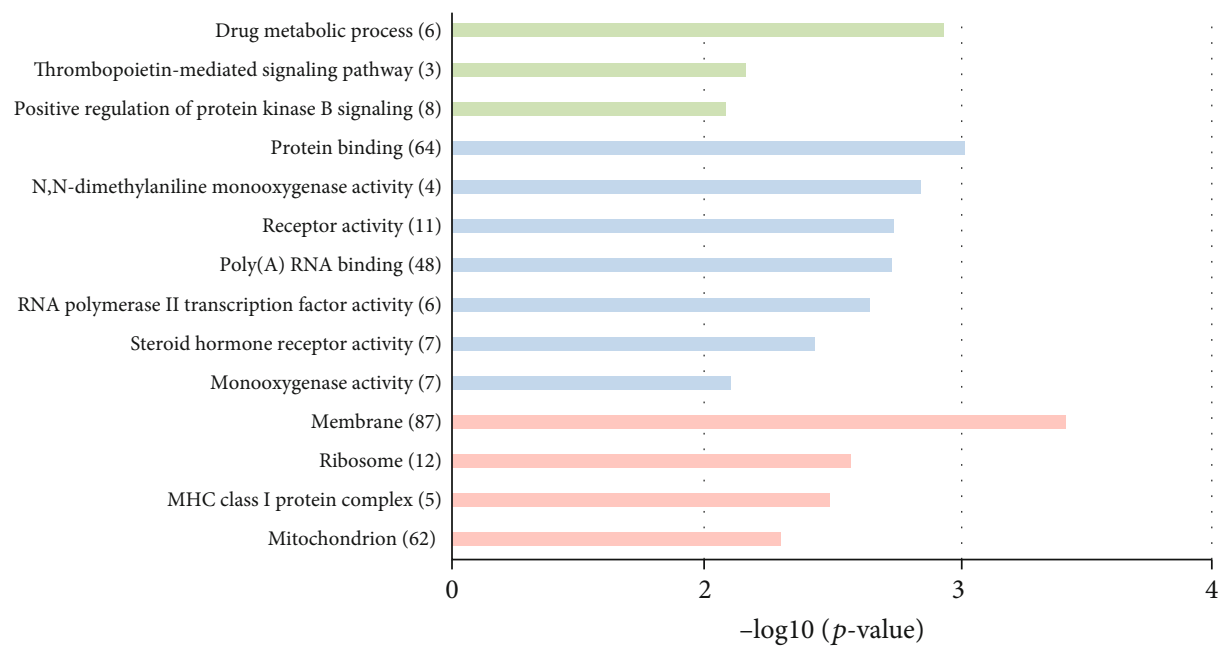

(b)

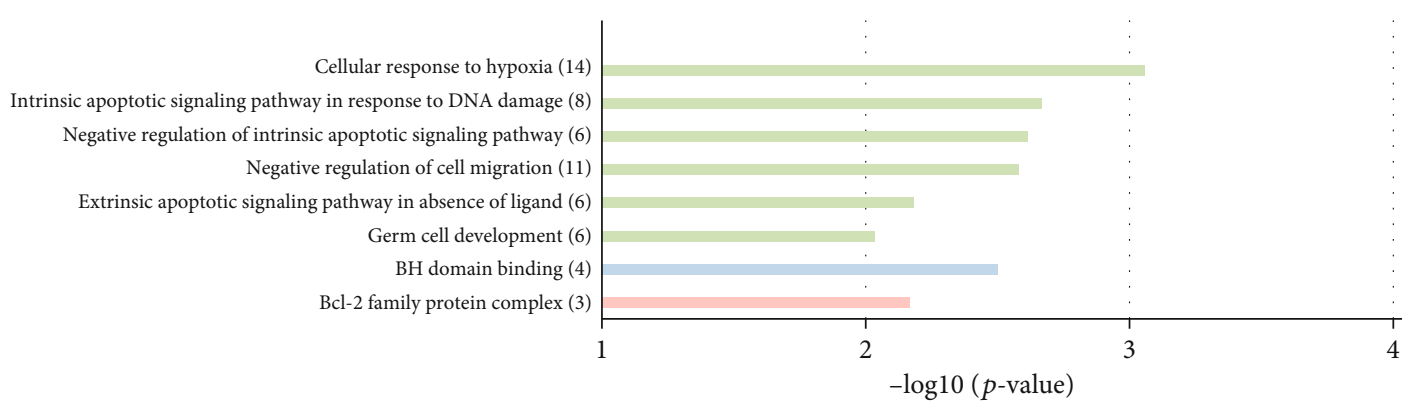

(c)

FiguRE 5: GO analysis of potential target genes of differentially expressed lncRNA NONMMUG000308 (a), NONMMUG001518 (b), and NONMMUG001518 (c). $y$-axis: GO terms of biological process, cellular component, and molecular function. The green columns indicate the biological process, the blue columns indicate molecular function, and the red columns indicate cellular component. The numbers of differentiated genes enriched in each term were displayed in brackets. $x$-axis: the negative logarithm of $p$ value.

showed that the top target overrepresented terms of lncRNAs included response to hypoxia, aging, and mitochondrion. A network of DE-lncRNAs and their potential target genes were established, and these DE-lncRNAs are named as Sancr 1,2 , and 3 , respectively.
As previous studies indicated that the hippocampal pathophysiological changes play important roles in the processes of POCD [13, 34], the hippocampus was selected for the present study. Cell culture [35], animal [36], and human [37] data indicate that inhaled anesthetics could accelerate 


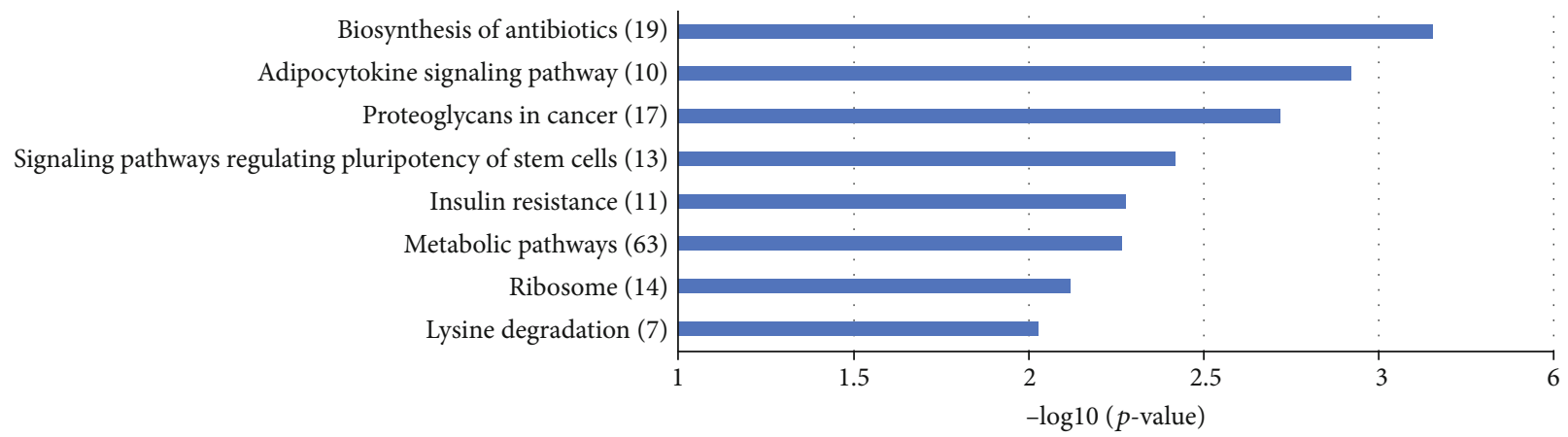

(a)

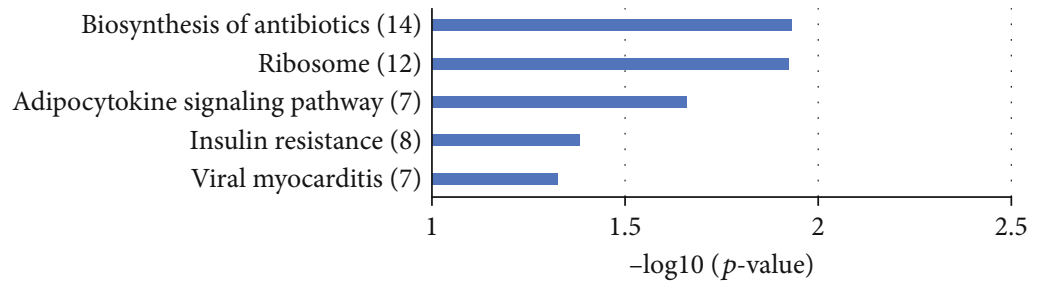

(b)

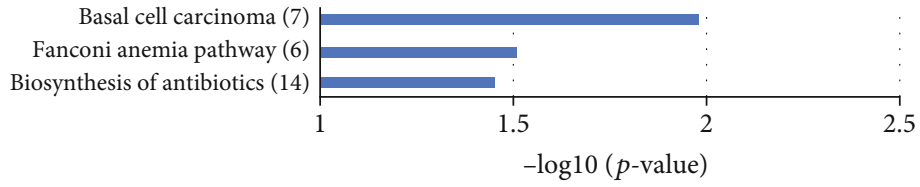

(c)

FIGURE 6: KEGG enrichment analysis of potential target genes of differentially expressed lncRNA NONMMUG000308 (a), NONMMUG001518 (b), and NONMMUG001518 (c). $y$-axis: KEGG terms descriptions. The numbers of differentiated genes enriched in each KEGG term were displayed in brackets. $x$-axis: the negative logarithm of $p$ value of different KEGG terms.

the development of aging and related neurodegenerations including apoptosis, $\mathrm{A} \beta$ generation, and tau phosphorylation. Combined with the present results, we infer that anesthesia also plays a role in the development of aging and related pathophysiological processes.

Accumulating evidences indicate the role of lncRNAs in the setting of oxidative stress, altered metabolism, and aging process, and they act as transcriptional factors or bind with target genes to exert functions. Several lincRNAs were differentially expressed in the hippocampus of both transgenic models and patients of $\mathrm{AD}[38,39]$, and the dysregulation of lincRNAs played key roles in the intricate regulation of CNS development and disorders [40, 41]. For example, lncRNA $B C 200$ is a transcript found in the brain with a function of inhibiting translation initiation. BC200 levels in cortical areas are reduced by above $60 \%$ in normal aging, in contrast, they are significantly upregulated in $\mathrm{AD}$ and paralleled with the deterioration of the disease [42]. IncRNA BACE1-AS concentrations were increased in $\mathrm{AD}$ subjects. It could change the secondary or tertiary structure of BACE1 through a mechanism affecting RNA duplex formation and so that increases its stability [17], and BACE1 is a crucial enzyme responsible for oligomer production in AD. Thus, we assumed that NONMMUG000308, NONMMUG001518, NONMMUG005150, and NONMMUG020388 regulate the expression level of target genes, which participates in the mechanism of sevoflurane-associated brain function modulation.
Since monitoring during anesthesia indicated that the rats did not suffer from hypoxia, and hippocampal MMP loss and ROS generation were observed during sevoflurane anesthesia, and emerging evidence revealed that HIFs expression can be induced by aberrant factors independent of oxygen levels [43, 44]. Hif3a and Epas1 (also called Hif2a) coding proteins are members of hypoxia-inducible factor (HIF) family, which are crucial modulators of transcriptional response to hypoxic stress $[45,46]$. We assumed that Sancrs regulated perioperative oxidative stress and related genes, such as Epas1 and Hif3a, transcriptional level, like lncRNA Neat2 [47]. Previous studies showed Hif3a mRNA expression can be regulated at the transcriptional level via hypoxia response elements [48] or posttranscriptional level via miRNA [49]. Gene Ontology annotations related to Hif3a include DNAbinding transcription factor activity and transcription coactivator activity, which are included in protein binding and consistent with the enriched terms in molecular function annotation of Sancr target genes.

The results indicated that $N f e 2 l 2, M t h f d 1 l, A k t 1$, and Atg 5 were possible targets of three DE-lncRNAs in metabolic pathways. In GO term aging, $\mathrm{Nfe} 2 \mathrm{l} 2$ was identified as a regulator of autophagy in degrading intraneuronal aggregates [50]. Nfe2l2 encoded transcription factor NRF2, which is the chief regulator to defense against stress in mammalian cells. NRF2 activation alleviates cognitive deficits of AD models through modulation of oxidative stress [51]. 


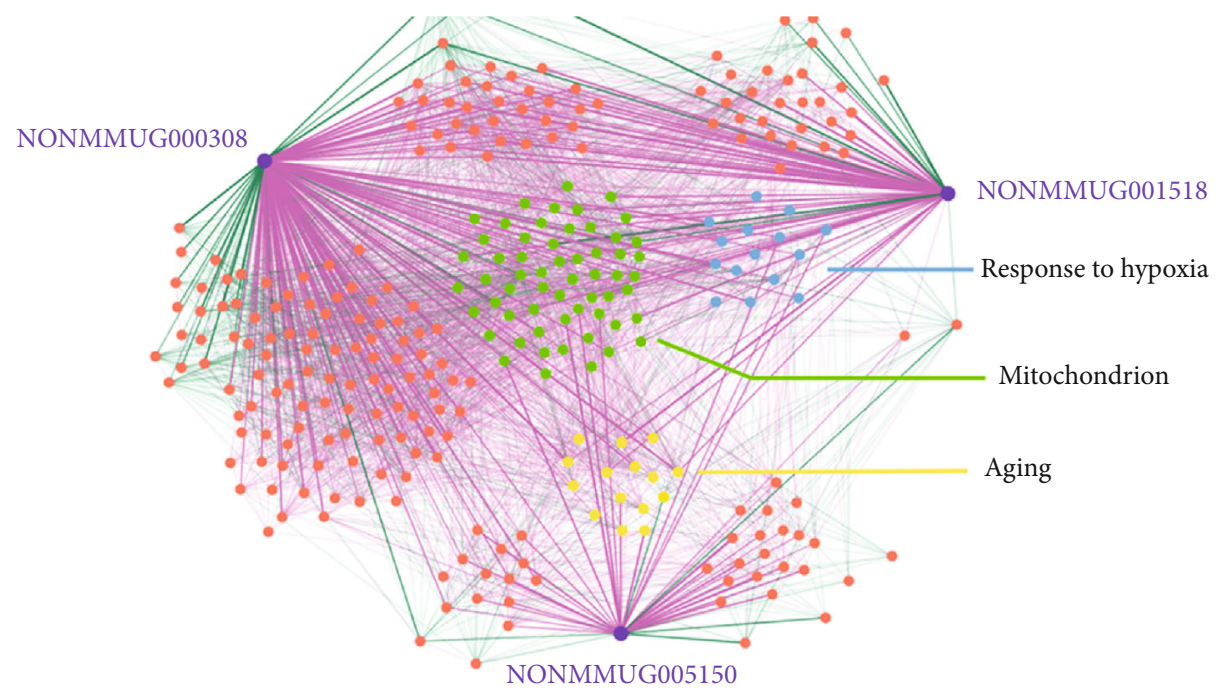

(a)

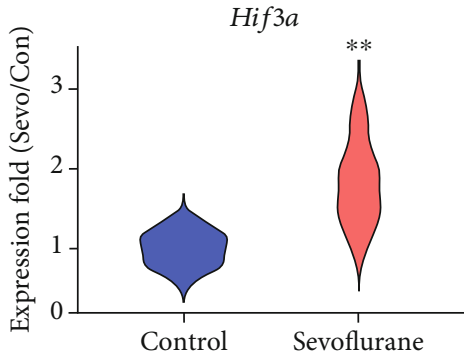

(b)

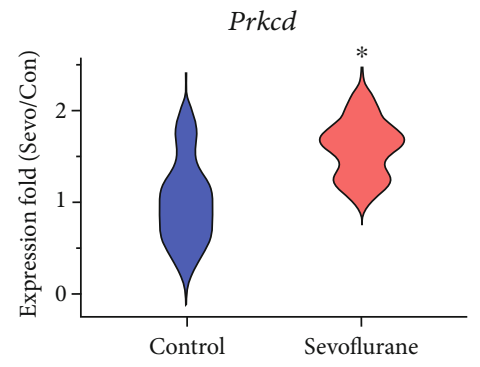

(c)

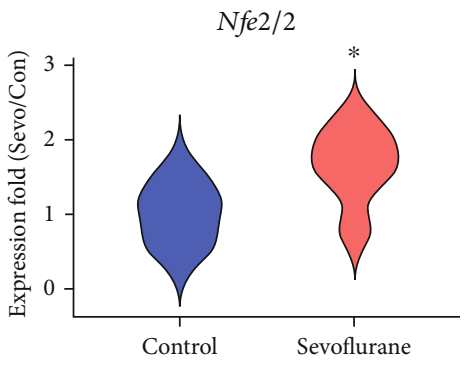

(d)

FiguRE 7: (a) LncRNA-mRNA coexpression network in the aged hippocampus after sevoflurane anesthesia. The purple nodes represent lncRNAs. The blue, green, yellow, and orange nodes represent gene-annotated GO terms response to hypoxia, mitochondrion, aging, and others. Coexpression pairs of lncRNA-mRNA whose Pearson correlation coefficient (PCC) $>0.85$ and mRNA-mRNA pairs whose PCC > 0.95 were connected by straight lines. Positive correlations are shown in red lines, and negative correlations in green lines. Lower transparency of edges means higher PCC. qPCR validation for representative differentially expressed genes (b) Hif $3 a$ from term hypoxia, (c) Prkcd from term mitochondrion, and (d) Nfe2l2 from term aging. $n=6,{ }^{*} p<0.05$, and ${ }^{* *} p<0.01$ indicated differentiated samples compared with the control condition.

MTHFD1L, an enzyme in the folate cycle that is transcriptionally activated by NRF2, plays an essential role in maintaining proper mitochondria function [52]. AKT1 could induce mitophagy due to reactive oxygen species [53]. Inhibition of mitochondrial permeability transition pore opening decreases ROS and increases MMP [54], and superoxide dismutase, glutathione peroxidase, and catalase levels could be affected during the process. The results also indicate that metabolism-related gene terms are affected by anesthesia. Combined with the present results, we propose that Sancrs interact with target genes and affect the metabolic process in the brain.

Sancrs were correlated to Prkcd expression, which was associated with the transcriptional regulation of p53 in response to DNA damage [55]. Dysregulation of DNA damage response is associated with neurodegenerative disorders [56], and the linkage between lncRNAs and neuron apoptosis has also been investigated in neurodegenerative diseases [57]. Both PKC $\delta$ and $\mathrm{p} 53$ are associated with the apoptotic mechanisms in the mitochondria through $\mathrm{Bcl}-2$ family proteins modulation and to provide mitochondrial outer membrane permeabilization [58]. Damage-induced lncRNA can create a feedback loop to amplify DNA damage signaling or interact with DNA damage RNAs through RNA-RNA pairs [59]. Thus, it is possible that Sancrs regulate genes like Prkcd or interact with other RNAs in this process and induced cell death. Our functional annotation results show that $\mathrm{BH}$ domain binding and the intrinsic mitochondrial apoptotic pathway Bcl-2 family complex are top significant enriched terms, which indicates Sancrs could be involved in intrinsic apoptosis in the hippocampus after anesthesia [60].

Our results show that Sancrs were highly coexpressed with Plat, and they could play a crucial role in perioperative ischemia-related diseases. Tissue plasminogen activator (encoded by Plat) is involved in the breakdown of blood clots. Genetic variants of Plat and plasminogen activator inhibitor type-1 combinations have been suggested to be the risk factors for stroke [61], and gene interactions could change the susceptibility of the disease. Disorders like amyotrophic lateral sclerosis, $\mathrm{AD}$, and Parkinson's disease have also been linked to reduced NRF2 levels [62, 63]. Combined with the results, we speculate that Sancrs bind with $\mathrm{Nfe} 2 \mathrm{l2}$, then accelerate stroke and neurodegenerative processes [64]. 


\section{Conclusion}

In the present investigation, we identified three DElncRNAs, Sancr 1, 2, and 3 in the hippocampus of aged rats after sevoflurane anesthesia. Sancrs regulate mitochondrial dysfunction and oxidative stress, aging-related metabolism alterations, DNA damage, and apoptosis, as well as stroke and neurodegenerative features in the hippocampus, which play roles in anesthesia-related cognitive function modulation during perioperative context. And similar to the mechanism of $\mathrm{AD}$, a complex network of deregulated and multitasking lncRNAs together interacts with these pathophysiological mechanisms. These results provide evidence for the lncRNA regulation network in anesthesia-related brain function modulation, which could be the understanding from an epigenetic perspective. However, detailed regulation pathways and correlated factors are unclarified. Further work should therefore investigate the accurate mechanisms and provide brain protection strategies through epigenetic perspective during the process.

\section{Data Availability}

The data of this study are openly available in Gene Expression Omnibus at https://www.ncbi.nlm.nih.gov/geo, with reference number GSE139220.

\section{Conflicts of Interest}

The authors report no conflict of interest.

\section{Authors' Contributions}

YQ performed the majority of the experiments, analyzed the data, and wrote the original draft of the manuscript. NY contributed to the experiments. LW contributed to the data acquisition and analysis. HL and MQ contributed to the experiment design and manuscript revision. XG contributed to the experiment design, data analysis, and manuscript revision. $\mathrm{CN}$ designed the project, supervised the experiments, drafted and revised the manuscript. All authors have read and approved the final manuscript.

\section{Acknowledgments}

This work was supported by the National Natural Science Foundation of China (Nos. 81771146, 81801070, and 81400869).

\section{References}

[1] Z. Xie and Z. Xu, "General anesthetics and $\beta$-amyloid protein," Progress in Neuro-Psychopharmacology \& Biological Psychiatry, vol. 47, pp. 140-146, 2013.

[2] T. G. Monk, B. C. Weldon, C. W. Garvan et al., "Predictors of cognitive dysfunction after major noncardiac surgery," Anesthesiology, vol. 108, no. 1, pp. 18-30, 2008.

[3] L. Evered, B. Silbert, D. S. Knopman et al., "Recommendations for the nomenclature of cognitive change associated with anaesthesia and surgery-2018," Anesthesiology, vol. 129, no. 5, pp. 872-879, 2018.

[4] K. J. Schenning, C. F. Murchison, N. C. Mattek, L. C. Silbert, J. A. Kaye, and J. F. Quinn, "Surgery is associated with ventricular enlargement as well as cognitive and functional decline," Alzheimers Dement, vol. 12, no. 5, pp. 590-597, 2016.

[5] A. M. Racine, T. G. Fong, Y. Gou et al., "Clinical outcomes in older surgical patients with mild cognitive impairment," Alzheimers Dement, vol. 14, no. 5, pp. 590-600, 2018.

[6] A. J. Davidson, N. Disma, J. C. de Graaff et al., "Neurodevelopmental outcome at 2 years of age after general anaesthesia and awake-regional anaesthesia in infancy (GAS): an international multicentre, randomised controlled trial," The Lancet, vol. 387, no. 10015, pp. 239-250, 2016.

[7] D. M. Norden and J. P. Godbout, "Review: microglia of the aged brain: primed to be activated and resistant to regulation," Neuropathology and Applied Neurobiology, vol. 39, no. 1, pp. 19-34, 2013.

[8] K. Schmitt, A. Grimm, A. Kazmierczak, J. B. Strosznajder, J. Gotz, and A. Eckert, "Insights into mitochondrial dysfunction: aging, amyloid- $\beta$, and tau-A deleterious trio," Antioxidants \& Redox Signaling, vol. 16, no. 12, pp. 1456-1466, 2012.

[9] Z. Li, N. Mo, L. Li et al., "Surgery-induced hippocampal angiotensin II elevation causes blood-brain barrier disruption via MMP/TIMP in aged rats," Frontiers in Cellular Neuroscience, vol. 10, p. 105, 2016.

[10] Z. Xu, Y. Dong, H. Wang et al., "Age-dependent postoperative cognitive impairment and Alzheimer-related neuropathology in mice," Scientific Reports, vol. 4, p. 3766, 2015.

[11] C. Ni, Z. Li, M. Qian, Y. Zhou, J. Wang, and X. Guo, "Isoflurane induced cognitive impairment in aged rats through hippocampal calcineurin/NFAT signaling," Biochemical and Biophysical Research Communications, vol. 460, no. 4, pp. 889-895, 2015.

[12] H. Miao, Y. Dong, Y. Zhang et al., "Anesthetic isoflurane or desflurane plus surgery differently affects cognitive function in Alzheimer's disease transgenic mice," Molecular Neurobiology, vol. 55, no. 7, pp. 5623-5638, 2018.

[13] Z. Wang, S. Meng, L. Cao, Y. Chen, Z. Zuo, and S. Peng, "Critical role of NLRP3-caspase-1 pathway in age-dependent isoflurane-induced microglial inflammatory response and cognitive impairment," Journal of Neuroinflammation, vol. 15, no. 1, p. 109, 2018.

[14] X. M. Li, F. Su, M. H. Ji et al., "Disruption of hippocampal neuregulin 1-ErbB4 signaling contributes to the hippocampusdependent cognitive impairment induced by isoflurane in aged mice," Anesthesiology, vol. 121, no. 1, pp. 79-88, 2014.

[15] M. Guttman, I. Amit, M. Garber et al., "Chromatin signature reveals over a thousand highly conserved large non-coding RNAs in mammals," Nature, vol. 458, no. 7235, pp. 223-227, 2009.

[16] P. Kapranov, J. Cheng, S. Dike et al., "RNA maps reveal new RNA classes and a possible function for pervasive transcription," Science, vol. 316, no. 5830, pp. 1484-1488, 2007.

[17] M. A. Faghihi, F. Modarresi, A. M. Khalil et al., "Expression of a noncoding RNA is elevated in Alzheimer's disease and drives rapid feed-forward regulation of beta-secretase," Nature Medicine, vol. 14, no. 7, pp. 723-730, 2008.

[18] J. R. Tollervey, T. Curk, B. Rogelj et al., "Characterizing the RNA targets and position-dependent splicing regulation by TDP-43," Nature Neuroscience, vol. 14, no. 4, pp. 452-458, 2011. 
[19] H. Yan, J. Rao, J. Yuan et al., "Long non-coding RNA MEG3 functions as a competing endogenous RNA to regulate ischemic neuronal death by targeting miR-21/PDCD4 signaling pathway," Cell Death \& Disease, vol. 8, no. 12, p. 3211, 2017.

[20] Y. Wang, M. Qian, Y. Qu et al., "Genome-wide screen of the hippocampus in aged rats identifies mitochondria, metabolism and aging processes implicated in sevoflurane anesthesia," Frontiers in Aging Neuroscience, vol. 12, p. 122, 2020.

[21] X. Q. Li, X. Z. Cao, J. Wang, B. Fang, W. F. Tan, and H. Ma, "Sevoflurane preconditioning ameliorates neuronal deficits by inhibiting microglial MMP-9 expression after spinal cord ischemia/reperfusion in rats," Molecular Brain, vol. 7, no. 1, p. 69, 2014.

[22] C. Ni, C. Li, Y. Dong, X. Guo, Y. Zhang, and Z. Xie, “Anesthetic isoflurane induces DNA damage through oxidative stress and p53 pathway," Molecular Neurobiology, vol. 54, no. 5, pp. 3591-3605, 2017.

[23] Y. Dong, G. Zhang, B. Zhang et al., “The common inhalational anesthetic sevoflurane induces apoptosis and increases betaamyloid protein levels," Archives of Neurology, vol. 66, no. 5, pp. 620-631, 2009.

[24] G. S. Julian, R. W. de Oliveira, J. C. Perry, S. Tufik, and J. R. Chagas, "Validation of housekeeping genes in the brains of rats submitted to chronic intermittent hypoxia, a sleep apnea model," PLoS One, vol. 9, no. 10, article e109902, 2014.

[25] N. Tanic, M. Perovic, A. Mladenovic, S. Ruzdijic, and S. Kanazir, "Effects of aging, dietary restriction and glucocorticoid treatment on housekeeping gene expression in rat cortex and hippocampus-evaluation by real time RT-PCR," Journal of Molecular Neuroscience, vol. 32, no. 1, pp. 38-46, 2007.

[26] T. J. Liu, B. Wang, Q. X. Li, X. L. Dong, X. L. Han, and S. B. Zhang, "Effects of microRNA-206 and its target gene IGF-1 on sevoflurane-induced activation of hippocampal astrocytes in aged rats through the PI3K/AKT/CREB signaling pathway," Journal of Cellular Physiology, vol. 233, no. 5, pp. 4294-4306, 2018.

[27] L. Chen, R. Dong, Y. Lu et al., "MicroRNA-146a protects against cognitive decline induced by surgical trauma by suppressing hippocampal neuroinflammation in mice," Brain, Behavior, and Immunity, vol. 78, pp. 188-201, 2019.

[28] T. Femenia, A. Gimenez-Cassina, S. Codeluppi et al., "Disrupted neuroglial metabolic coupling after peripheral surgery," The Journal of Neuroscience, vol. 38, no. 2, pp. $452-464,2018$.

[29] J. S. Morey, J. C. Ryan, and F. M. Van Dolah, "Microarray validation: factors influencing correlation between oligonucleotide microarrays and real-time PCR," Biol Proced Online, vol. 8, no. 1, pp. 175-193, 2006.

[30] I. Martinez-Reyes, L. P. Diebold, H. Kong et al., “TCA cycle and mitochondrial membrane potential are necessary for diverse biological functions," Molecular Cell, vol. 61, no. 2, pp. 199-209, 2016.

[31] E. Gottlieb, S. M. Armour, M. H. Harris, and C. B. Thompson, "Mitochondrial membrane potential regulates matrix configuration and cytochrome c release during apoptosis," Cell Death and Differentiation, vol. 10, no. 6, pp. 709-717, 2003.

[32] L. A. Sena and N. S. Chandel, "Physiological roles of mitochondrial reactive oxygen species," Molecular Cell, vol. 48, no. 2, pp. 158-167, 2012.

[33] X. Guo, L. Gao, Q. Liao et al., "Long non-coding RNAs function annotation: a global prediction method based on bi- colored networks," Nucleic Acids Research, vol. 41, no. 2, p. e35, 2013.

[34] R. Yirmiya and I. Goshen, "Immune modulation of learning, memory, neural plasticity and neurogenesis," Brain, Behavior, and Immunity, vol. 25, no. 2, pp. 181-213, 2011.

[35] Y. Zhen, Y. Dong, X. Wu et al., "Nitrous oxide plus isoflurane induces apoptosis and increases beta-amyloid protein levels," Anesthesiology, vol. 111, no. 4, pp. 741-752, 2009.

[36] Y. Dong, X. Wu, Z. Xu, Y. Zhang, and Z. Xie, “Anesthetic isoflurane increases phosphorylated tau levels mediated by caspase activation and Abeta generation," PLoS One, vol. 7, no. 6 , article e39386, 2012.

[37] B. Zhang, M. Tian, H. Zheng et al., "Effects of anesthetic isoflurane and desflurane on human cerebrospinal fluid $\mathrm{A} \beta$ and $\tau$ level," Anesthesiology, vol. 119, no. 1, pp. 52-60, 2013.

[38] M. Magistri, D. Velmeshev, M. Makhmutova, and M. A. Faghihi, "Transcriptomics profiling of Alzheimer's disease reveal neurovascular defects, altered amyloid- $\beta$ homeostasis, and deregulated expression of long noncoding RNAs," Journal of Alzheimer's Disease, vol. 48, no. 3, pp. 647-665, 2015.

[39] D. Y. Lee, J. Moon, S. T. Lee et al., "Distinct expression of long non-coding RNAs in an Alzheimer's disease model," Journal of Alzheimer's Disease, vol. 45, no. 3, pp. 837-849, 2015.

[40] S. Y. Ng, L. Lin, B. S. Soh, and L. W. Stanton, "Long noncoding RNAs in development and disease of the central nervous system," Trends in Genetics, vol. 29, no. 8, pp. 461-468, 2013.

[41] N. N. Parikshak, V. Swarup, T. G. Belgard et al., "Genomewide changes in lncRNA, splicing, and regional gene expression patterns in autism," Nature, vol. 540, no. 7633, pp. 423-427, 2016.

[42] E. Mus, P. R. Hof, and H. Tiedge, "Dendritic BC200 RNA in aging and in Alzheimer's disease," Proceedings of the National Academy of Sciences of the United States of America, vol. 104, no. 25, pp. 10679-10684, 2007.

[43] S. Mohlin, A. Hamidian, K. von Stedingk et al., "PI3KmTORC2 but not PI3K-mTORC1 regulates transcription of HIF2A/EPAS1 and vascularization in neuroblastoma," Cancer Research, vol. 75, no. 21, pp. 4617-4628, 2015.

[44] M. Y. Koh, M. Gagea, T. Sargis et al., "A new HIF$1 \alpha /$ RANTES-driven pathway to hepatocellular carcinoma mediated by germline haploinsufficiency of SART1/HAF in mice," Hepatology, vol. 63, no. 5, pp. 1576-1591, 2016.

[45] A. J. Majmundar, W. J. Wong, and M. C. Simon, "Hypoxiainducible factors and the response to hypoxic stress," Molecular Cell, vol. 40, no. 2, pp. 294-309, 2010.

[46] F. J. Gonzalez, C. Xie, and C. Jiang, "The role of hypoxiainducible factors in metabolic diseases," Nature Reviews. Endocrinology, vol. 15, no. 1, pp. 21-32, 2018.

[47] B. Zhang, G. Arun, Y. S. Mao et al., "The lncRNA Malat1 is dispensable for mouse development but its transcription plays a cis-regulatory role in the adult," Cell Reports, vol. 2, no. 1, pp. 111-123, 2012.

[48] M. Heikkila, A. Pasanen, K. I. Kivirikko, and J. Myllyharju, "Roles of the human hypoxia-inducible factor (HIF)-3 $\alpha$ variants in the hypoxia response," Cellular and Molecular Life Sciences, vol. 68, no. 23, pp. 3885-3901, 2011.

[49] C. M. Gits, P. F. van Kuijk, J. C. de Rijck et al., "MicroRNA response to hypoxic stress in soft tissue sarcoma cells: microRNA mediated regulation of HIF3alpha," BMC Cancer, vol. 14, p. 429, 2014. 
[50] M. Pajares, N. Jimenez-Moreno, A. J. Garcia-Yague et al., "Transcription factor NFE2L2/NRF2 is a regulator of macroautophagy genes," Autophagy, vol. 12, no. 10, pp. 1902-1916, 2016.

[51] A. I. Rojo, M. Pajares, P. Rada et al., "NRF2 deficiency replicates transcriptomic changes in Alzheimer's patients and worsens APP and TAU pathology," Redox Biology, vol. 13, pp. 444-451, 2017.

[52] D. Lee, I. M. Xu, D. K. Chiu et al., "Folate cycle enzyme MTHFD1L confers metabolic advantages in hepatocellular carcinoma," The Journal of Clinical Investigation, vol. 127, no. 5, pp. 1856-1872, 2017.

[53] J. L. Larson-Casey, J. S. Deshane, A. J. Ryan, V. J. Thannickal, and A. B. Carter, "Macrophage Akt1 kinase-mediated mitophagy modulates apoptosis resistance and pulmonary fibrosis,” Immunity, vol. 44, no. 3, pp. 582-596, 2016.

[54] Y. Zhang, Z. Xu, H. Wang et al., "Anesthetics isoflurane and desflurane differently affect mitochondrial function, learning, and memory," Annals of Neurology, vol. 71, no. 5, pp. 687698, 2012.

[55] N. Dashzeveg and K. Yoshida, "Crosstalk between tumor suppressors $\mathrm{p} 53$ and $\mathrm{PKC} \delta$ : execution of the intrinsic apoptotic pathways," Cancer Letters, vol. 377, no. 2, pp. 158-163, 2016.

[56] S. Matt and T. G. Hofmann, "The DNA damage-induced cell death response: a roadmap to kill cancer cells," Cellular and Molecular Life Sciences, vol. 73, no. 15, pp. 2829-2850, 2016.

[57] M. J. Millan, "Linking deregulation of non-coding RNA to the core pathophysiology of Alzheimer's disease: an integrative review," Progress in Neurobiology, vol. 156, pp. 1-68, 2017.

[58] N. Dashzeveg and K. Yoshida, "Cell death decision by p53 via control of the mitochondrial membrane," Cancer Letters, vol. 367, no. 2, pp. 108-112, 2015.

[59] F. Michelini, S. Pitchiaya, V. Vitelli et al., "Damage-induced lncRNAs control the DNA damage response through interaction with DDRNAs at individual double-strand breaks," Nature Cell Biology, vol. 19, no. 12, pp. 1400-1411, 2017.

[60] N. Joza, S. A. Susin, E. Daugas et al., "Essential role of the mitochondrial apoptosis-inducing factor in programmed cell death," Nature, vol. 410, no. 6828, pp. 549-554, 2001.

[61] M. S. Babu, T. S. Prabha, S. Kaul et al., "Association of genetic variants of fibrinolytic system with stroke and stroke subtypes," Gene, vol. 495, no. 1, pp. 76-80, 2012.

[62] A. Jan, B. Jansonius, A. Delaidelli et al., "eEF2K inhibition blocks A $\beta 42$ neurotoxicity by promoting an NRF2 antioxidant response," Acta Neuropathologica, vol. 133, no. 1, pp. 101-119, 2017.

[63] F. Sivandzade, S. Prasad, A. Bhalerao, and L. Cucullo, "NRF2 and NF- $\triangle \mathrm{B}$ interplay in cerebrovascular and neurodegenerative disorders: molecular mechanisms and possible therapeutic approaches," Redox Biology, vol. 21, p. 101059, 2019.

[64] N. Kubben, W. Zhang, L. Wang et al., "Repression of the antioxidant NRF2 pathway in premature aging," Cell, vol. 165, no. 6, pp. 1361-1374, 2016. 